\title{
A Framework for Patient-Specific Physics-Based Simulation and Interactive Visualization of Cardiac Dynamics
}

\author{
Wei-te Lin and Richard A. Robb \\ Mayo Foundation/Clinic, Rochester, Minnesota 55905
}

\section{Introduction}

Characterization of cardiac motion can be derived from a pre-operatively acquired image sequence of an entire cardiac cycle. However, changes in the patient's cardiac morphology and physiology during and after interventions will not be reflected in the pre-acquired data. What is needed is a faithful, patient-specific deformable model of the heart that can be used on-line with interventional cardiac procedures. Diffusionencoded magnetic resonance imaging provides data for more precise modeling of a specific heart. A computer simulation which integrates fiber information with a physics-based mechanical model and a simulated electrical conduction model has been developed to realistically mimic cardiac dynamics. Such a model can ultimately be used to guide treatment interventions and to predict surgical outcomes.

\section{Methods}

Implementation of the simulation system includes four major components:

\subsection{Reconstruction of Regional Fiber Orientation}

Multiple MR images of an excised heart were acquired with diffusion encoded in six directions. The high resolution images were interpolated to produce $1 \mathrm{~mm}^{3}$ voxels. Regional myocardial fiber orientation is defined for each voxel by the eigenvector corresponding to the largest eigenvalue of the diffusion tensor composed by the six MR images [1].

\subsection{Cardiac Electrical Conduction Simulation}

The conduction is simulated using a cellular automata model [2]. Each muscle voxel is modeled to have three conduction states: the resting state, the activation state, and the refractory state. Each muscle voxel performs shortening when activated by neighboring muscle voxels.

\subsection{Cardiac Muscle Contraction Simulation}

A contraction model is constructed for each muscle voxel. The muscle cell network is constructed by arranging muscle voxels in a 3-D grid with grid points connected by simulated springs (see Fig. 1). Muscle contraction is simulated by systematically performing shortening of individual muscle voxels. This leads to deformation of the entire heart.

\subsection{Interactive Visualization}

The surface model is deformed and displayed using a free-form deformation method. The surface model of the heart, reconstructed from the MR image, is embedded in the 3-D grid that is used to form the muscle cell network. Muscle voxel shortening results 
in displacements of the grid points, which deform the nearby space and cause the embedded surface model to deform.

(a)

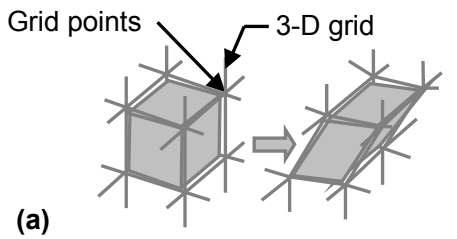

(b)

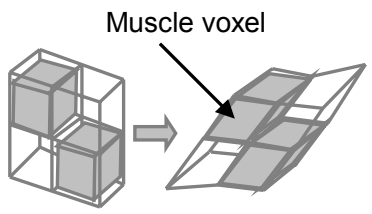

Fig. 1. Simulated muscle shortening. (a) Muscle voxel shortening results in deformation of the grid. (b) Muscle voxels are connected by a 3-D grid to form a muscle cell network.

\section{Results}

Fig. 2 illustrates results of applying the model to a sheep heart. The images represent geometry changes of the left ventricle during systole.

(a)

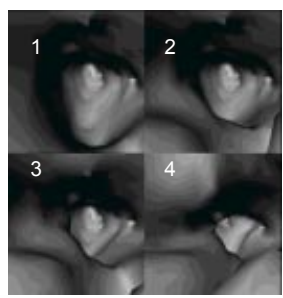

(b)

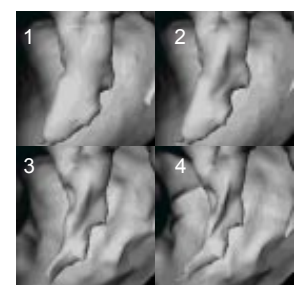

Fig. 2. Simulated heartbeat of the sheep heart. (a) Figure shows four time points during systole, viewing from inside the left ventricle toward the apex. (b) Deformation of the left ventricle.

\section{Discussion}

Integrating regional fiber information with simulated electrical conduction and muscle contraction models provides realistic simulation of cardiac motion. With improving diffusion MR techniques, it will be possible to acquire high-resolution in vivo patient heart images for patient-specific muscle fiber mappings and models. For validation, images of an entire cardiac cycle acquired using high-speed CT scanners, 3-D ultrasound, and techniques such as MR tagging can be used for quantitative comparison. The extensible framework allows such factors as fluid dynamics of blood flow, reception of and response to signals from the central nervous system, and various genetic and acquired anomalies to be readily incorporated into the simulation.

\section{References}

1. Hsu, E.W., Muzikant, A.L., Matulevicius, S.A., Penland, R.C. and Henriquez, C.S.: Magnetic resonance myocardial fiber-orientation mapping with direct histological correction. Am. J. Physiol. 274 (1998) H1627-H1634.

2. Saxberg, B.E.H., and Cohen, R.J.: Cellular automata models of cardiac conduction. Theory of Heart: Biomechanics, Biophysics, and Nonlinear Dynamics of Cardiac Function. Edited by Glass, L., Hunter, P., and McCulloch, A., Springer Verlag, 1991. 